\title{
Precision Attitude Control for the BETTII Balloon-Borne Interferometer
}

The Balloon Experimental Twin Telescope for Infrared Interferometry (BETTII) is an 8-meter baseline far-infrared interferometer to fly on a high altitude balloon. Operating at wavelengths of 30-90 microns, BETTII will obtain information on science targets at angular resolutions down to less than half an arcsecond. This requires attitude control at a level of less than a tenth of an arcsecond. We have designed a precision attitude determination and multi-stage control system providing precision pointing stability to the level required for the far-infrared instrument to perform its spatial/spectral interferometry open-loop. We present key aspects of the design and its development status.

Full:

The Balloon Experimental Twin Telescope for Infrared Interferometry (BETTII) is an 8-meter baseline far-infrared interferometer to fly on a high altitude balloon. Operating at wavelengths of 30-90 microns, BETTII will obtain spatial and spectral information on science targets at angular resolutions down to less than half an arcsecond, a capability unmatched by other far-infrared facilities. This requires attitude control at a level of less than a tenth of an arcsecond, a great challenge for a lightweight balloon-borne system. We have designed a precision attitude determination system to provide gondola attitude knowledge at a level of 2 milliarcseconds at rates up to $100 \mathrm{~Hz}$, with accurate absolute attitude determination at the half arcsecond level at rates of up to $10 \mathrm{~Hz}$. A multi-stage control system involving rigid body motion and tip-tilt-piston correction provides precision pointing stability to the level required for the far-infrared instrument to perform its spatial/spectral interferometry in an open-loop control. We present key aspects of the design of the attitude determination and control and its development status.

\section{Biography:}

Dr. Benford received the Ph.D. in Astrophysics from Caltech in research on the development and use of bolometer instruments for detecting distant galaxies. He has been at NASA's Goddard Space Flight Center in Greenbelt, Maryland since 1999. He has pursued research in far-infrared bolometric detectors, long wavelength observations of star forming regions and ultraluminous galaxies, and cryogenic instrumentation for space observatories. His primary research focus is in observational extragalactic astronomy and cosmology and developing ultrasensitive instruments for far-infrared and submillimeter astronomy. He is currently the Chief Scientist of NASA's Cosmic Origins Program Office and the Deputy Mission Scientist for the Wide-Field Infrared Survey Explorer (WISE).

Additional Authors:

Dale Fixsen 
Stephen Rinehart

Keywords:

Precision Pointing Balloon-borne telescope Infrared interferometry Attitude determination Attitude control 